\title{
Convergent Validity of a Physical Activity Questionnaire against Objectively Measured Physical Activity in Adults: The Cardiovascular Risk in Young Finns Study
}

\author{
Mirja Hirvensalo ${ }^{*}$, Costan G. Magnussen ${ }^{2,3}$, Xiaolin Yang ${ }^{4}$, Risto Telama ${ }^{1}$, Ilkka Heinonen ${ }^{5,6,7}$, \\ Nina Hutri-Kähönen ${ }^{8}$, Kasper Salin'1, Jorma Viikari², olli T. Raitakari², Tuija H. Tammelin ${ }^{4}$ \\ ${ }^{1}$ Faculty of Sport and Health Sciences, University of Jyväskylä, Jyväskylä, Finland \\ ${ }^{2}$ Research Centre of Applied and Preventive Cardiovascular Medicine and Departments of Clinical Physiology and Internal \\ Medicine, University of Turku and Turku University Central Hospital, Turku, Finland \\ ${ }^{3}$ Menzies Research Institute Tasmania, University of Tasmania, Hobart, Australia \\ ${ }^{4}$ LIKES Research Centre for Physical Activity and Health, Jyväskylä, Finland \\ ${ }^{5}$ Turku PET Centre, Turku University Hospital, University of Turku, Turku, Finland \\ ${ }^{6}$ Department of Clinical Physiology and Nuclear Medicine, University of Turku, Turku, Finland \\ ${ }^{7}$ Division of Experimental Cardiology, Thoraxcenter, Erasmus MC, University Medical Center Rotterdam, Rotterdam, \\ the Netherlands \\ ${ }^{8}$ Department of Pediatrics, University of Tampere and Tampere University Hospital, Tampere, Finland \\ Email: ^mirja.hirvensalo@jyu.fi
}

How to cite this paper: Hirvensalo, M., Magnussen, C. G., Yang, X. L., Telama, R., Heinonen, I., Hutri-Kähönen, N., Salin, K., Viikari, J., Raitakari, O. T., \& Tammelin, T. H. (2017). Convergent Validity of a Physical Activity Questionnaire against Objectively Measured Physical Activity in Adults: The Cardiovascular Risk in Young Finns Study. Advances in Physical Education, 7, 457-472. https://doi.org/10.4236/ape.2017.74038

Received: August 29, 2017

Accepted: November 19, 2017

Published: November 22, 2017

Copyright $\odot 2017$ by authors and Scientific Research Publishing Inc. This work is licensed under the Creative Commons Attribution International License (CC BY 4.0).

http://creativecommons.org/licenses/by/4.0/

\begin{abstract}
Background: Traditionally, a self-reported questionnaire has been a cost-effective method of gathering information about physical activity (PA). An objective measurement, such as the use of a pedometer, can be used to validate the findings of a PA questionnaire in a large population. Objective: The study objective was to determine the convergent validity of a PA questionnaire against objectively measured PA in adults obtained with the use of a pedometer. Methods: Data from the Cardiovascular Risk in Young Finns Study (YFS) were collected from 1853 participants aged 30 - 45 years. The participants completed a self-reported questionnaire that included items on leisure time, commuting and habitual PA. PA was expressed as leisure-time physical activity index (PAI) and metabolic equivalent hours/week (METh/wk). The participants wore a pedometer for seven consecutive days and used it to record their total daily and aerobic steps. Results: There was a low to moderate association between the self-reported questionnaire and pedometer measurements regarding both total steps and aerobic steps taken during leisure time and commuting PA. An association was not observed between pe-
\end{abstract}


dometer data and habitual PA. Of the individual items in the questionnaire, questions that described the frequency of PA and the duration of vigorous PA correlated the most strongly with the pedometer values obtained for total and aerobic steps $(r=0.28-0.44, p \leq 0.010)$. Conclusions: These findings suggest that the YFS PA questionnaire showed acceptable convergent validity in assessing, in particular, exercise-type PA in an adult population.

\section{Keywords}

Questionnaire, Pedometer, Adults, Total Steps, Aerobic Steps, Physical Activity

\section{Introduction}

Information on population-based and community-dwelling physical activity (PA) has predominantly been collected through the administration of self-report questionnaires. Questionnaires provide a cost-effective means of monitoring the content and sub-domains of PA in large population studies (Lamonte \& Ainsworth, 2001; van Poppel, Chinapaw, Mokkink, van Mechelen, \& Terwee, 2010; Shephard, 2003; Terwee, Mokkink, van Poppel, Chinapaw, van Mechelen, \& de Vet, 2010). Recently, however, the use of an objective measure, such as a pedometer, has been the focus of PA studies because it permits more accurate quantification. Pedometers have proven useful in monitoring and quantifying everyday activities because they are able to measure incidental PA (Cocker, Cardon, \& De Bourdeaudhuii, 2007; Tudor-Locke, Williams, Reis, \& Pluto, 2002). Even though there are some issues related to pedometers (e.g., they are not capable of being used in certain activities), they have been shown to provide objectively measured PA, which is relatively well correlated with aerobic capacity (Michaud, Cauderay, Narring, \& Schultz, 2002). On the other hand, some pedometers might undercount steps and they are not as accurate as accelerometers (Crouter, Schneider, \& Bassett, 2005). At the same time, it is important not to dismiss the rich contextual information provided by questionnaires (Tudor-Locke et al., 2002). Objective measures are increasingly being used to determine the reliability and validity of different PA measurements obtained from various PA questionnaires and when making comparisons between them (Boon, Hamlin, Steel, \& Ross, 2010; Helmerhorst, Brage, Warren, Besson, \& Ekelund, 2012).

In a comprehensive review by Helmerhorst et al. (2012), PA questionnaires were validated with the use of objective measures. It was concluded that self-reported questionnaires remain an important research method in large-scale studies. They are a practical way of assessing PA and when stratifying risk and appraising disease etiology. Most PA questionnaires are designed to measure multiple dimensions of PA and provide estimates of time spent on activities of various levels of intensity. They may also be able to rank individuals according to intensity levels of reported activity. However, a PA questionnaire adminis- 
tered to one population cannot be systematically extrapolated to other populations, age groups, ethnic groups or geographical regions. Gender differences are not typically addressed in research, but some questions may be more suitable to men than to women, owing to cultural differences in PA behavior, for example. Gender differences in leisure time, commuting and occupational PA were identified in Finland (Borodulin, Harald, Jousilahti, Laatikainen, Männistö, \& Vartiainen, 2016). Women were physically active more frequently than men were, but the degree of PA intensity was higher among men.

The median validity of self-reported PA questionnaires has been found to be low (Landis \& Koch, 1977). Correlation coefficients range from 0.30 to 0.39 for existing questionnaires and from 0.25 to 0.41 for new ones (Helmerhorst et al., 2012). A validity study was performed for both the New Zealand Physical Activity Questionnaire and the International Physical Activity Questionnaire. There was a low correlation with the ActiGraph ${ }^{\circledast}$ data for time spent in moderate-intensity PA $(r=0.19-0.30)$ and moderate- and vigorous-intensity PA ( $r=$ 0.30 - 0.32; Boon et al., 2010). Other than correlation coefficients, respondent classification into tertiles (percentage of agreement and gross misclassification) has been used to assess the validity of questionnaires. It has been found that this is a valid and reliable instrument to do so (Stel, Smit, Plujim, Visser, Deeg, \& Lips, 2004).

The objective of the current study was to assess the convergent validity of the Cardiovascular Risk in Young Finns Study (YFS) PA questionnaire against the measurements obtained for step count using a pedometer. The same population has been investigated by the YFS longitudinal research project for 31 years and includes regular follow-ups. It is important that the validity of the questionnaire and the historical data are evaluated using an objective measure to ensure the reliability of data for future projects. These results may be useful to other similar PA studies. PA questionnaire data have been previously used to provide estimates of energy expenditure as metabolic equivalent hours/week (METh/wk) values (Stel et al., 2004), in cardiovascular risk analysis (Yang, Telama, Hirvensalo, Mattson, Viikari, \& Raitakari, 2008) and to grade individuals according to PA during their life course (Telama, Yang, Leskinen, Kankaanpää, Hirvensalo, \& Tammelin, 2014). The concept of PA in the YFS is mainly based on PA as a lifestyle. Lifestyle in this context refers to physical activity choices made by people in terms of their ability, such as leisure-time PA, voluntary PA, active commuting and habitual activities. The PA questions have been structured to measure multiple PA dimensions by reporting on frequency, intensity, duration and activity. Pedometers were also used to assess daily PA in the YFS in 2007.

This article assesses convergent validity by comparing the self-report YFS PA questionnaire with pedometer step counts in a sample of 1853 men and women aged 30 to 45 years. Total daily steps, aerobic steps and aerobic minutes were collected and compared with self-reported leisure-time PA, commuting PA, and habitual PA, expressed as leisure-time PA index (PAI) and as METh/wk values. 
The results are presented separately for men and women.

\section{Methods}

\subsection{Participants}

The Cardiovascular Risk in Young Finns Study (YFS; Åkerblom, Viikari, Uhari, Räsänen, Byckling, Louhivuori et al., 1985; Raitakari, Juonala, \& Rönnemaa, 2008) is an ongoing follow-up study undertaken on six cohorts born in 1962, $1965,1968,1971,1974$ and 1977. In 1980, these participants $(N=3596)$ were randomly selected from the Finnish national register of the study areas: Helsinki, Kuopio, Oulu, Tampere, and Turku and their surrounding communities. They were followed up on in 1983, 1986, 1989, 1992, 2001, and 2007. The 2007 follow-up was performed between October 2007 and February 2008, with 2204 participants aged $30,33,36,39,42$, and 45 years. Table 1 presents the mean age of the participants, $37.8 \pm 5.0$ years (women) and $37.6 \pm 5.1$ years (men). Seventy-nine percent of the participants were employed (manual workers $31 \%$, non-manual workers at low level 27\%, and non-manual workers at high level $42 \%)$. Three percent of the participants were unemployed, $8 \%$ were students, $2 \%$ were disabled and unable to work, and $8 \%$ remained home with their children or

Table 1. Characteristics of the study participants $(N=1853)$.

\begin{tabular}{|c|c|c|c|c|c|c|}
\hline & \multicolumn{3}{|c|}{ Women } & \multicolumn{3}{|c|}{ Men } \\
\hline & \multicolumn{3}{|c|}{ Mean $\pm s$} & \multicolumn{3}{|c|}{ Mean $\pm s$} \\
\hline Age, years & \multicolumn{3}{|c|}{$37.8 \pm 5.0$} & \multicolumn{3}{|c|}{$37.6 \pm 5.1$} \\
\hline Education, $\leq 12$ years & \multicolumn{3}{|c|}{$15.8 \pm 3.5$} & \multicolumn{3}{|c|}{$15.0 \pm 3.4^{*}$} \\
\hline Body mass index, $\mathrm{kg} / \mathrm{m}^{2}$ & \multicolumn{3}{|c|}{$24.3 \pm 4.9$} & \multicolumn{3}{|c|}{$26.5 \pm 4.2^{*}$} \\
\hline Waist circumference, $\mathrm{cm}$ & \multicolumn{3}{|c|}{$83.4 \pm 12.2$} & \multicolumn{3}{|c|}{$93.9 \pm 11.8^{*}$} \\
\hline Pedometer values & Median & 25th & 75th & Median & 25th & 75th \\
\hline Total steps/day & 7518 & 5797 & 9553 & 6761 & 4981 & 8791 \\
\hline Aerobic steps/day & 1902 & 598 & 3422 & 729 & 0 & 2072 \\
\hline Aerobic minutes/week & 114 & 34 & 203 & 46 & 0 & 127 \\
\hline Aerobic step intensity, steps/min & 115 & 108 & 122 & 110 & 101 & 118 \\
\hline \multicolumn{7}{|l|}{$\begin{array}{l}\text { Self-reported PA: Leisure-time physical } \\
\text { activity index (PAI) }\end{array}$} \\
\hline PAI, range 5 - 15 & 9 & 8 & 10 & 9 & 7 & 10 \\
\hline \multicolumn{7}{|l|}{$\begin{array}{l}\text { Self-reported PA: MET-index and its } \\
\text { components }\end{array}$} \\
\hline Leisure-time PA, METh/wk & 10.0 & 2.0 & 25.0 & $8.0^{\star}$ & 0.5 & 50.0 \\
\hline Commuting PA, METh/wk & 0 & 0 & 7 & 0 & 0 & 0 \\
\hline Habitual PA, METh/wk & 12.3 & 2 & 37.7 & 13.4 & 6.9 & 24.4 \\
\hline Total PA, METh/wk ${ }^{\mathrm{a}}$ & 45.3 & 26.8 & 74.3 & $35.7^{\star}$ & 16.1 & 35.7 \\
\hline
\end{tabular}

Data are mean ( \pm standard deviation) for age, years of education, body mass index, waist circumference, and median (25th, 75th percentiles) for pedometer and physical activity indexes; ${ }^{\text {a }}$ um of Leisure-time PA, Commuting PA, and Habitual PA; METh/wk = Metabolic equivalent hours per week; ${ }^{\star}$ Mean value significant different than women, $p<0.001$. 
did not specify an answer (Hirvensalo, Telama, Schmidt, Tammelin, Yang, Magnussen et al., 2011).

Although all were invited to wear pedometers, 1874 participants completed the pedometer study. Those individuals who completed the pedometer study were more often women $(p<0.001)$ and had a higher level of education $(p=$ 0.002). The level of self-rated PA did not differ significantly from those who did not participate in the pedometer study (Hirvensalo et al., 2011). After removing those with missing or incomplete data, information on 1853 participants was included in this study. The study participants gave written informed consent while the study protocol was reviewed and approved by the ethics committees of the participating universities (Decision number 533/2006).

\subsection{Study Protocol}

PA and participation in sports were measured by means of a self-report questionnaire that was administered individually in connection with a medical examination (Raitakari et al., 2008). At the same time, participants received an Omron Walking Style One (HJ-152R-E) pedometer.During waking hours, they attached the pedometer to their waistband or belt in the same position for seven consecutive days and maintained a pedometer log.

In the pedometer logs participants recorded the time they removed the pedometer and, at the end of the day, the number of steps taken as shown on the display. Participants also indicated whether the day of pedometer wear was representative of their usual PA or, if not, the reason for the exceptions. Participants engaged in their typical activities and removed the pedometer only while bathing or swimming. Participants could report comments and problems about their pedometer use in the pedometer log and could contact the study personnel if needed. On the eighth day, participants were instructed to send their pedometer with the log to the study centers using a padded mail bag in a self-addressed, stamped envelope that was provided to all participants.

\subsection{Objective Measures of PA by Pedometer}

The Omron pedometer collects aerobic steps and minutes in addition to total steps. Aerobic steps are those taken during activities that last for at least ten minutes without interruption at a pace of 60 or more steps per minute. The mean intensity of aerobic step activity is determined by dividing the aerobic step count by aerobic minutes. In a previous study, we compared Omron Walking Style

One pedometers with the steps measured by ActiGraph accelerometers (GT1M) in a subsample of 45 participants for six to seven successive days (total of 304 days). The Spearman's rank correlation coefficient was $0.966(p<0.001$; Mansikkaniemi et al., 2012).

Those who had at least four monitoring days with at least eight hours of pedometer wear time were included in all the analyses. Sickness or injury status, exceptional step counts recorded as an atypical day, or problems with pedometer 
use were imputed by the mean of other days. Days of non-wear, or days with exceptionally high step counts that participants identified as atypical, were imputed with the mean of other days. Participants recorded several reasons for daily nonparticipation or interruption to pedometer wear. The main reasons were a lost $(n=52)$ or broken pedometer $(n=23)$, illness $(n=30)$, or other reasons such as an untypical day $(n=22)$.

Total steps and aerobic steps were used as continuous variables in descriptive and correlation analysis and as categorical variables in comparisons with other measurements. Total steps were classified in tertiles as follows: Low $\leq 6038$ steps, Medium 6039 - 8432 steps, and High $\geq 8433$ steps per day. The level of aerobic steps were classified into three categories according to the duration and intensity of ambulatory activity: 1) No aerobic steps = those who had zero aerobic steps; 2) Low aerobic steps $=1-150$ minutes of aerobic steps per week and those who did not belong in Category 3; and 3) High aerobic steps = those with at least 150 minutes aerobic steps per week with an intensity of 100 steps per minute or those with 75 minutes aerobic steps per week with an intensity of at least 130 steps per minute (Haskell, Lee, Pate, Powell, Blair, Franklin et al., 2007; Marshall, Levy, Tudor-Locke, Kolkhorst, Wooten, Ji et al., 2009).

\subsection{Physical Activity Questionnaire}

Leisure time, commuting and habitual PA were assessed using the YFS PA questionnaire. The answers were converted into leisure-time PAI scores and expressed as MET hours per week, which described the PA in terms of energy expenditure (see Appendix 1).

\subsubsection{Leisure-Time PA Index (PAI)}

The questionnaire consisted of five questions concerning the intensity of PA, the frequency of PA, hours per week spent on PA, average duration of one PA session, and participation in organized PA. The original questions are presented in Appendix 1 as well as coding for calculating a leisure-time PA index (PAI; Telama, Yang, Viikari, Välimäki, Wanne, \& Raitakari, 2005).

\subsubsection{PA Expressed as METh/Wk}

Leisure-time $P A$. Leisure-time PA was converted into METh/wk by multiplying the frequency, intensity and duration of PA. The intensity of PA was estimated based on the Compendium of Physical Activities (Ainsworth, Haskell, Whitt, Irwin, Swartz, Strath et al., 2000). The original questions and coefficients used for calculating leisure-time PA to METh/wk are presented in Appendix 1.

Commuting PA. The mode of commuting and commuting distance were asked by separate questions for summer and winter conditions, and coded $1=$ personal car or carpool, 2 = public transport, $3=$ on foot, $4=$ by bicycle. Distance travelled to work on foot or by bicycle was reported in kilometers and converted into minutes using $5 \mathrm{~km} / \mathrm{h}$ as an average speed for walking and 15 $\mathrm{km} / \mathrm{h}$ for biking. When calculating METh/wk values, we used intensity values of 
3.5 MET for walking and 4 MET for biking (Ainsworth et al., 2000). Walking to and from the bus was also taken into account. See the original question in Appendix 1 .

Habitual $P A$. The participants were asked how many hours per month they performed habitual light-, moderate- or heavy-intensity activities at home. The answers were converted into METh/wk values by dividing monthly hours by 4.3 and then multiplying these amounts with intensity values of 2 MET for light activities, 3 MET for moderate and 4 MET for heavy activities.

Total PA. As a sum of LTPA, commuting PA and habitual PA, we calculated a total PA MET index, describing overall weekly PA as METh/wk.

\subsection{Statistical Analyses}

Means and standard deviations were used to describe age, years of education, body mass index (BMI) and waist circumference. Sex differences in variables were analyzed using the t-test. Median and interquartile ranges, 25th and 75th percentile values, were calculated for skewed distributions of step values and PA variables. The associations between pedometer step counts (total steps and aerobic steps) and items on the PA questionnaire were first studied by calculating Spearman correlation coefficients stratified by sex.

In line with a previous study by Stel et al. (2004), each respondent was classified into three categories based on leisure-time PAI, which varied from 5 to 15 . The three PAI level classifications (Low: $<8$ points (23.9\% of participants), Medium: 8 - 9 points (42.5\% of participants) and High: $\geq 10$ points (33.6\% of participants) were compared with three-level classifications using total and aerobic steps from the pedometer. The percentage of agreement and misclassification between categories of the questionnaire's leisure-time PAI with total and aerobic step counts was calculated. Gross misclassification was defined as respondents who were classified into the highest tertile on the questionnaire's leisure-time PAI and into the lowest tertile on total or aerobic steps, or vice-versa.

In addition, convergent validity analyses were performed by comparing means and standard deviations of daily total steps and aerobic steps and weekly minutes of aerobic steps and their intensity (steps per minute) in three categories of leisure-time PAI. All statistical analyses were performed using SPSS Statistics for Windows, version 24.0.

\section{Results}

Women had $7824 \pm 2925$ and men $7089 \pm 2774$ total steps/day. Women took $2327 \pm 2143$ and men $1420 \pm 1809$ aerobic steps/day. Median as well as 25 th and 75th percentile values for step values and PA variables are presented in Table 1. The median LTPA was not significantly different in women and men but the median total PA (METh/wk) was higher for women than it was for men $(p<$ 0.001). While total PA (METh/wk) was made up of predominantly leisure-time and habitual PA, more than three-quarters of the population reported no com- 
muting PA. The mean value of commuting PA was $5.4 \pm 11.1 \mathrm{METh} / \mathrm{wk}$ in women and 3.6 $\pm 11.0 \mathrm{METh} / \mathrm{wk}$ in men.

Table 2 shows that PA variables from the self-reported questionnaire were significantly and positively correlated with total steps and aerobic steps, except for habitual PA. Among women and men, correlations with the PAI were stronger for aerobic steps than for total steps. The strongest correlations with aerobic steps were observed for the question "How often do you engage in sport or physical activity so that you get out of breath and sweat?" Again, the strongest correlations were observed for aerobic steps. As with the PAI, correlations of total PA (METh/wk) with aerobic steps were higher than with total steps. Correlations of commuting PA expressed as METh/wk were low but positive with total and aerobic steps. The correlation coefficients of habitual PA METh/wk with total steps and aerobic steps were not significant.

Based on the comparison of total steps and PAI levels, 39.3\% of the respondents were classified to the same category, $26.9 \%$ of the respondents were classified to a higher category and $33.7 \%$ to a lower category than total steps tertiles (Table 3). Gross misclassification (a two-category difference) between the classification of the results (PAI) relating to the questionnaire and those for the pedometer steps occurred for $13.6 \%$ of the participants. Better agreement was achieved with the comparison of the aerobic step category results.

In the aerobic step and LTPA comparison, $43.6 \%$ of the respondents were classified into the same category and gross misclassification occurred for $7.9 \%$ of

Table 2. Spearman's rank order correlations for the association between physical activity (PA) questions and pedometer values.

\begin{tabular}{ccccc}
\hline \multirow{2}{*}{ PA questions and indices } & \multicolumn{4}{c}{ Pedometer values } \\
\cline { 2 - 5 } & \multicolumn{2}{c}{ Total steps } & Aerobic steps \\
\cline { 2 - 5 } & $\begin{array}{c}\text { Women } \\
(\mathrm{n}=1058)\end{array}$ & $\begin{array}{c}\text { Men } \\
(\mathrm{n}=790)\end{array}$ & $\begin{array}{c}\text { Women } \\
(\mathrm{n}=1058)\end{array}$ & $\begin{array}{c}\text { Men } \\
(\mathrm{n}=790)\end{array}$ \\
\hline $\begin{array}{c}\text { Self-reported PA: Leisure-time physical activity } \\
\text { index (PAI) and its components }\end{array}$ & & & & \\
Intensity of PA & 0.05 & $0.10^{*}$ & $0.11^{* *}$ & $0.20^{* *}$ \\
Frequency of sport or PA & $0.31^{* *}$ & $0.28^{* *}$ & $0.44^{* *}$ & $0.39^{* *}$ \\
Hours per week of sport or PA & $0.30^{* *}$ & $0.28^{* *}$ & $0.41^{* *}$ & $0.35^{* *}$ \\
Duration of one session of sport of PA & $0.20^{* *}$ & $0.21^{* *}$ & $0.25^{* *}$ & $0.22^{* *}$ \\
Participation in organized PA & $0.13^{* *}$ & 0.08 & $0.24^{* *}$ & 0.24 \\
PAI, range 5 - 15 & $0.25^{* *}$ & $0.25^{* *}$ & $0.31^{* *}$ & $0.30^{* *}$ \\
Self-reported PA: MET-index and its components & & & & \\
Leisure-time PA, METh/wk & $0.264^{* *}$ & $0.285^{* *}$ & $0.366^{* *}$ & $0.406^{* *}$ \\
Commuting PA, METh/wk & $0.192^{* *}$ & $0.213^{* *}$ & $0.196^{* *}$ & $0.282^{* *}$ \\
Habitual PA, METh/wk & -0.013 & -0.013 & -0.075 & -0.121 \\
Total PA, METh/wk & $0.200^{*}$ & $0.168^{* *}$ & $0.205^{* *}$ & $0.137^{* *}$ \\
\hline
\end{tabular}

${ }^{\text {aS }}$ um of Leisure-time PA, Commuting PA, and Habitual PA; ${ }^{* *}$ Correlation is significant at the 0.01 level (2-tailed); ${ }^{*}$ Correlation is significant at the 0.05 level (2-tailed). 
Table 3. Comparison of Questionnaire leisure-time physical activity index (PAI) tertile groups (low, medium, high) with total step tertile groups and aerobic steps categories. Data are proportions of all participants (\%).

\begin{tabular}{|c|c|c|c|c|c|c|c|c|c|}
\hline \multirow[b]{3}{*}{ Total step levels ${ }^{\mathrm{a}}$} & \multicolumn{9}{|c|}{ PAI categories ${ }^{c}$} \\
\hline & \multicolumn{3}{|c|}{ All $(n=1805)$} & \multicolumn{3}{|c|}{ Women $(n=1037)$} & \multicolumn{3}{|c|}{$\operatorname{Men}(n=768)$} \\
\hline & Low & Medium & High & Low & Medium & High & Low & Medium & High \\
\hline Low & 11.2 & 7.0 & 5.7 & 8.6 & 13.2 & 6.6 & 14.7 & 14.8 & 9.8 \\
\hline Medium & 13.9 & 14.5 & 14.2 & 7.6 & 14.9 & 11.3 & 6.3 & 14.1 & 12.8 \\
\hline High & 7.9 & 11.9 & 13.6 & 5.4 & 17.6 & 14.9 & 6.1 & 9.6 & 11.8 \\
\hline Agreement ${ }^{\mathrm{d}}$ & & & 39.3 & & & 38.4 & & & 40.6 \\
\hline \multirow[t]{2}{*}{ Misclassification $^{\mathrm{e}}$} & & & 13.6 & & & 12.0 & & & 15.9 \\
\hline & \multicolumn{3}{|c|}{ Total $(n=1818)$} & \multicolumn{3}{|c|}{ Women $(n=1042)$} & \multicolumn{3}{|c|}{ Men $(n=776)$} \\
\hline Aerobic step levels ${ }^{\mathrm{b}}$ & Low & Medium & High & Low & Medium & High & Low & Medium & High \\
\hline No aerobic steps & 7.9 & 7.4 & 4.3 & 5.5 & 4.9 & 2.1 & 11.2 & 10.8 & 7.2 \\
\hline Low & 12.4 & 21.3 & 14.9 & 11.2 & 22.6 & 13.5 & 14.0 & 19.6 & 16.8 \\
\hline High & 3.6 & 13.8 & 14.4 & 4.9 & 18.1 & 17.2 & 1.8 & 7.9 & 10.7 \\
\hline Agreement & & & 43.6 & & & 45.3 & & & 41.5 \\
\hline Misclassification & & & 7.9 & & & 7.0 & & & 9.0 \\
\hline
\end{tabular}

${ }^{a}$ Total steps in tertiles, Low $\leq 6038$, Medium 6039 - 8432 and High $\geq 8433$ steps; ${ }^{b}$ Aerobic steps' weekly duration and intensity: No aerobic steps (19.6\% of participants), Low $=$ all others with aerobic steps (48.6\% of participants), High $=$ those who had 150 minutes of aerobic steps in a week with an intensity of 100 steps/min and those who recorded at least 75 minutes aerobic steps in a week with an intensity of 130 steps/min (31.7\% of participants); 'Leisure-time physical activity index (PAI, varied $5-15$ ) was divided into three categories: Low $\leq 8$ points ( $23.9 \%$ of participants), Medium $=8-9$ points $(42.5 \%$ of participants) and High $\geq 10$ points (33.6\% of participants); ${ }^{\mathrm{d}}$ Agreement $=$ sum $\%$ of the responses in exactly same tertile; ${ }^{\mathrm{e}}$ Misclassification = proportion of respondents who were classified into the highest tertile on the questionnaire leisure-time physical activity index (PAI).

respondents. Women had a somewhat higher agreement percentage than men $\operatorname{did}(45.3 \%$ vs. $41.5 \%)$.

The means of daily total steps varied significantly between the three categories of the PAI in women and men (Table 4). Women that reported high PA according to the PAI took an average of 8625 steps/day and accumulated on average over 1600 more steps/day than those in the low PAI category and over 900 more steps/day than women in the medium PAI category $(p<0.001)$. In men, the differences between categories were similar but the total amount of steps in every category was 800 - 1000 steps lower than it was for women. In addition, daily aerobic steps, weekly aerobic minutes, and the intensity of aerobic steps tended to increase from those in the low category of the PAI to those in the high category group.

\section{Discussion}

Low to moderate levels of convergent validity were demonstrated with the use of the self-reported YFS PA questionnaire when those results were compared with the objective pedometer data. The validity coefficients for the PA measurements 
Table 4. Means of total steps, aerobic steps and weekly minutes of aerobic steps and their intensity (steps/min) in three categories of leisure-time physical activity index (PAI).

\begin{tabular}{|c|c|c|c|c|c|c|c|c|c|c|}
\hline \multirow{3}{*}{ Step variables } & \multicolumn{5}{|c|}{ Women } & \multicolumn{5}{|c|}{ Men } \\
\hline & \multicolumn{5}{|c|}{ PAI } & \multicolumn{5}{|c|}{ PAI } \\
\hline & $N$ & $\begin{array}{l}\text { Low } \\
\text { mean }\end{array}$ & $\begin{array}{c}\text { Medium } \\
\text { mean }\end{array}$ & $\begin{array}{l}\text { High } \\
\text { mean }\end{array}$ & $P$ & $n$ & $\begin{array}{l}\text { Low } \\
\text { mean }\end{array}$ & $\begin{array}{c}\text { Medium } \\
\text { mean }\end{array}$ & $\begin{array}{l}\text { High } \\
\text { mean }\end{array}$ & $P$ \\
\hline Total steps/day & 1037 & 6994 & 7729 & 8625 & $<0.001$ & 765 & 6261 & 7075 & 7781 & $<0.001$ \\
\hline Aerobic steps/day & 1037 & 1494 & 2270 & 3022 & $<0.001$ & 765 & 734 & 1376 & 2033 & $<0.001$ \\
\hline Aerobic steps, min/week & 1034 & 91 & 135 & 171 & $<0.001$ & 764 & 47 & 84 & 116 & $<0.001$ \\
\hline Intensity, aerobic steps/min & 908 & 111 & 114 & 119 & $<0.001$ & 546 & 105 & 110 & 115 & $<0.001$ \\
\hline
\end{tabular}

ranged from $r=0.20$ to 0.30 (total steps) and $r=0.20$ to 0.44 (aerobic steps), similar to what has typically been reported for such questionnaires (Boon et al., 2010; Helmerhorst et al., 2012). In the review by Helmerhorst et al. (2012), the median validity correlation coefficient was $r=0.25$ - 0.41 for the PA questionnaires.

Several questions were included in the YFS to capture different aspects of a physically active lifestyle. The sum of the PAI index scores, based on five PA questions (in which scores $<8$ were considered low active, scores $8-9$ were medium active, and scores $\geq 10$ were high active), was associated with the average number of daily pedometer steps, with correlation coefficients ranging from $r=$ 0.25 to 0.31 . The comprehensiveness and relevance of the questionnaire should be evaluated against the setting, construct, and recall period as well as the purpose and target population (Terwee et al., 2010). The provision of in-depth detail on frequency, duration, intensity and included activities is appropriate for a variety of reasons in longitudinal studies such as the YFS, including being able to assess the impact of different components of childhood physical activity on adulthood PA and other health behavior, being able to track PA and for the purpose of cardiovascular and other disease risk analysis.

PA has been broadly defined as "all bodily actions produced by the contraction of skeletal muscle that increase energy expenditure above basal level" (Butte, Ekelund, \& Westerterp, 2012). We attempted to get a broad understanding of PA by evaluating leisure-time, commuting and habitual activities in the questionnaire and by converting them into METh/wk values that express PA in terms of energy expenditure. Leisure-time PA and commuting PA expressed in $\mathrm{METh} / \mathrm{wk}$ appeared to be more in line with the objective measures of total and aerobic steps than with those for habitual PA. Both the pedometer measures and the questionnaire are, however, only estimations of energy consumption. In addition, some people are unwilling to wear a pedometer or any other device for a week, as required by the research protocol. Thus, it may be easier to simply complete a questionnaire for participants.

LTPA was converted into METh/wk by multiplying the frequency, intensity and duration of LTPA. The correlation coefficients were at the same low to 
moderate level as the coefficient with the questions, and better than the PAI, indicating that the LTPA METh/wk variable aligns better with the pedometer measure than does the LTPA. The weaker coefficients for the PAI might be a result of including information on the type of sport participation. The commuting MET index correlated positively with steps, as shown by the correlation coefficient. The coefficients with total steps were lower than with aerobic steps, and higher among women than they were among men. Women's commuting was especially associated with aerobic steps, which we believe is a result of their general commuting practices, with $16.7 \%$ of women commuting by walking compared with $7.9 \%$ of men (not shown in results). The correlations with habitual PA (METh/wk) were low and not statistically significant. Pedometers may not be ideal for measuring PA in habitual activities that often include activities that can be performed while standing or sitting and thus would not contribute to overall step counts.

\section{Activity Level Comparisons}

Our results show that the YFS leisure-time PAI estimates of low, medium, or high level PA were different from those of the pedometer's low, medium and high step levels (misclassification 13.6\%, total gross misclassification $60.7 \%$, agreement 39.3\%). However, the results are comparable with a previous study (Stel et al., 2004) in the Netherlands in which they validated the LASA Physical Activity Questionnaire with pedometer scores. The comparison between three-level classifications of leisure-time PAI and aerobic step categories had somewhat better agreement than with total step categories. Aerobic steps reflect exercise activities better than total steps because they are continuous, lasting for at least ten minutes without interruption.

A number of factors may contribute to the lack of convergent validity in the measurement of habitual PA. First, the assessment period of the YFS PA questionnaire was not of similar length and did not cover the same period as the seven-day pedometer study. The study participants answered their typical PA in general, but the seven-day pedometer log gathered steps from the week when they used a pedometer. Activities could differ between typical weeks and the week that was reported. Furthermore, in this study week and weekend activities were not separated. This may have led to an underestimation of the validity of the questionnaire. The questionnaire may also reflect more general, year-round PA than the pedometer does. In theory, the questionnaire has no seasonal variation, but pedometer measures are dependent on weather and temperature, which can differ by season and region. Second, pedometers do not quantify cycling, skiing, and swimming and a questionnaire may not capture the small amounts of habitual activity, such as those associated with activities that did not in general correlate with our pedometer data. In addition, the pedometer did not capture all activity performed by our study population. For example, $43 \%$ of men and $38 \%$ of women reported activities that the pedometer could not measure (e.g., 
skiing, cycling, swimming, skating, water aerobics, home aerobics). However, sensitivity analyses showed that correlations were essentially similar after excluding these participants from the analyses (not shown in results).

\section{Conclusion}

Since no gold standard criterion method exists to record the time spent at various intensities (Macfarlane, Lee, Ho, Chan, \& Chan, 2006; Schmidt, Freedson, \& Chasan-Taber, 2003), we can only conclude that the questionnaires and pedometers both measure PA adequately but in different manners. It is unrealistic to expect high agreement between an instrument that has exact PA cut points and the less precise self-report questionnaire. In this study, aerobic steps provided a relevant estimate for moderate- and high-level PA when duration and intensity could be considered. It could be that relationships with health outcomes are stronger when using a metric such as aerobic steps than when using one such as total steps.

Our results suggest that the YFS PA questionnaire is an acceptably valid subjective measure of lifestyle PA in Finnish adults and the practical advantages of its self-reporting will assure its continued use. In a large community-based population, the questionnaire was easy to use and the self-report method provided contextual information on the type of PA that is not available from pedometer or other motion sensors.

\section{Acknowledgements}

The Young Finns Study has been financially supported by the Academy of Finland: grants 286284, 134309 (Eye), 126925, 121584, 124282, 129378 (Salve), 117787 (Gendi) (ST), and 41071 (Skidi); the Social Insurance Institution of Finland; Competitive State Research Financing of the Expert Responsibility area of Kuopio, Tampere and Turku University Hospitals (grant X51001); Juho Vainio Foundation (ST); Paavo Nurmi Foundation; Finnish Foundation for Cardiovascular Research; Finnish Cultural Foundation (ST); Tampere Tuberculosis Foundation; Emil Aaltonen Foundation; Yrjö Jahnsson Foundation; Signe and Ane Gyllenberg Foundation and Diabetes Research Foundation of Finnish Diabetes Association.

\section{References}

Ainsworth, B. E., Haskell, W. L., Whitt, M. C., Irwin, M. L., Swartz, A. M., Strath, S. J. et al. (2000). Compendium of Physical Activities: An Update of Activity Codes and MET Intensities. Medicine \& Science in Sports \& Exercise, 32, S498-S504. https://doi.org/10.1097/00005768-200009001-00009

Åkerblom, H. K., Viikari, J., Uhari, M., Räsänen, L., Byckling, T., Louhivuori, K. et al. (1985). Atherosclerosis Precursors in Finnish Children and Adolescents. I. General Description of the Cross-Sectional Study of 1980, and an Account of the Children's and Families' State of Health. Acta Paediatrica, 74, S49-S63.

https://doi.org/10.1111/j.1651-2227.1985.tb10082.x 
Boon, R. M., Hamlin, M. J., Steel, G. D., \& Ross, J. J. (2010). Validation of the New Zealand Physical Activity Questionnaire (NZPAQ-LF) and the International Physical Activity Questionnaire (IPAQ-LF) with Accelerometry. British Journal of Sports Medicine, 44, 741-746. https://doi.org/10.1136/bjsm.2008.052167

Borodulin, K., Harald, K., Jousilahti, P., Laatikainen, T., Männistö, S., \& Vartiainen, E. (2016). Time Trends in Physical Activity from 1982 to 2012 in Finland. Scandinavian Journal in Medicine \& Science in Sports, 26, 93-100. https://doi.org/10.1111/sms.12401

Butte, N. F., Ekelund, U., \& Westerterp, K. R. (2012). Assessing Physical Activity Using Wearable Monitors: Measures of Physical Activity. Medicine \& Science in Sports \& Exercise, 44, S5-S12. https://doi.org/10.1249/MSS.0b013e3182399c0e

Cocker, K. D., Cardon, G., \& De Bourdeaudhuij, I. (2007). Pedometer-Determined Physical Activity and Its Comparison with the International Physical Activity Questionnaire in a Sample of Belgian Adults. Research Quarterly for Exercise and Sports, 78, 429-437. https://doi.org/10.1080/02701367.2007.10599443

Crouter, S. E., Schenider, P. L., \& Bassett, D. R. (2005). Spring-Leveled versus Piezo-Electric Pedometer Accuracy in Overweight and Obese Adults. Medicine \& Science in Sports \& Exercise, 37, 1673-1679. https://doi.org/10.1249/01.mss.0000181677.36658.a8

Haskell, W., Lee, I., Pate, R., Powell, K. E., Blair, S. N., Franklin, B. A. et al. (2007). Physical Activity and Public Health: Updated Recommendation for Adults from the American College of Sports Medicine and the American Heart Association. Medicine \& Science in Sports \& Exercise, 39, 1423-1434. https://doi.org/10.1249/mss.0b013e3180616b27

Helmerhorst, H. H. J. F., Brage, S., Warren, J., Besson, H., \& Ekelund, U. (2012). A Systematic Review of Reliability and Objective Criterion-Related Validity of Physical Activity Questionnaires. International Journal of Behavioral Nutrition and Physical Activity, 9, 103. https://doi.org/10.1186/1479-5868-9-103

Hirvensalo, M., Telama, R., Schmidt, M. D., Tammelin, T., Yang, X., Magnussen, C. et al. (2011). Daily Steps among Finnish Adults: Variation by Age, Gender, and Socioeconomic Position. Scandinavian Journal of Public Health, 39, 669-677. https://doi.org/10.1177/1403494811420324

Lamonte, M. J., \& Ainsworth, B. E. (2001). Quantifying Energy Expenditure and Physical Activity in the Context of Dose Response. Medicine and Science in Sports \& Exercise, 33, S370-S378. https://doi.org/10.1097/00005768-200106001-00006

Landis, J. R., \& Koch, G. G. (1977). The Measurement of Observer Agreement for Categorical Data. Biometrics, 33, 159-174. https://doi.org/10.2307/2529310

Macfarlane, D. J., Lee, C. C. Y., Ho, E. Y. K., Chan, K. L., \& Chan, D. (2006). Convergent Validity of Six Methods to Assess Physical Activity in Daily Life. Journal of Applied Physiology, 1, 1328-1334. https://doi.org/10.1152/japplphysiol.00336.2006 http://jap.physiology.org/content/101/5/1328.short

Mansikkaniemi, K., Juonala, M., Taimela, S., Hirvensalo, M., Telama, R., Huupponen, R. et al. (2012). Cross-Sectional Associations between Physical Activity and Selected Coronary Heart Disease Risk Factors in Young Adults. The Cardiovascular Risk in Young Finns Study. Annals of Medicine, 44, 733-744. https://doi.org/10.3109/07853890.2011.590146

Marshall, S. J., Levy, S. S., Tudor-Locke, C. E., Kolkhorst, F. W., Wooten, K. M., Ji, M. et al. (2009). Translating Physical Activity Recommendations into a Pedometer-Based Step Goal: 3000 Steps in 30 Minutes. American Journal of Preventive Medicine, 36, 410-415. https://doi.org/10.1016/j.amepre.2009.01.021 
Michaud, P. A., Cauderay, M., Narring, F., \& Schultz, Y. (2002). Assessment of Physical Activity with a Pedometer and Its Relationship with $\mathrm{V}=2 \max$ among Adolescents in Switzerland. Sozial-und Päventivmedizin, 47, 107-115.

https://doi.org/10.1007/BF01318392

Raitakari, O. T., Juonala, M., \& Rönnemaa, T. (2008). Cohort Profile: The Cardiovascular Risk in Young Finns Study. International Journal of Epidemiology, 37, 1220-1226. https://doi.org/10.1093/ije/dym225

Schmidt, M. D., Freedson, P. S., \& Chasan-Taber, L. (2003). Estimating Physical Activity Using the CSA Accelerometer and a Physical Activity Log. Medicine \& Science in Sports \& Exercise, 35, 1605-1611. https://doi.org/10.1249/01.MSS.0000084421.97661.17

Shephard, R. J. (2003). Limits to the Measurement of Habitual Physical Activity by Questionnaires. British Journal of Sports Medicine, 37, 197-206.

https://doi.org/10.1136/bjsm.37.3.197

Stel, V. S., Smit, J. H., Pluijm, S. M., Visser, M., Deeg, D. J. H., \& Lips, P. (2004). Comparison of the LASA Physical Activity Questionnaire with a 7-Day Diary and Pedometer. Journal of Clinical Epidemiology, 57, 252-258. https://doi.org/10.1016/j.jclinepi.2003.07.008

Telama, R., Yang, X., Leskinen, E., Kankaanpää, A., Hirvensalo, M., Tammelin, T. et al. (2014). Tracking Physical Activity from early Childhood through Youth into Adulthood over 27 Years. Medicine \& Science in Sports \& Exercise, 46, 955-962. https://doi.org/10.1249/MSS.0000000000000181

Telama, R., Yang, X., Viikari, J., Välimäki, I., Wanne, O., \& Raitakari, O. T. (2005). Physical Activity from Childhood to Adulthood-A 21-Year Tracking Study. American Journal of Preventive Medicine, 28, 267-273. https://doi.org/10.1016/j.amepre.2004.12.003

Terwee, C. B., Mokkink, L. B., van Poppel, M. N., Chinapaw, M. J. M, van Mechelen, W., \& De Vet, H. C. W. (2010). Qualitative Attributes and Measurement Properties of Physical Activity Questionnaires: A Checklist. Sports Medicine, 40, 525-537. https://doi.org/10.2165/11531370-000000000-00000

Tudor-Locke, C., Williams, J. E., Reis, J. P., \& Pluto, D. (2002). Utility of Pedometers for Assessing Physical Activity: Construct Validity. Sports Medicine, 34, 281-291. https://doi.org/10.2165/11531370-000000000-00000

van Poppel, M. N., Chinapaw, M. J., Mokkink, L. B., van Mechelen, W., \& Terwee, C. B. (2010). Physical Activity Questionnaires for Adults: A Systematic Review of Measurement Properties. Sports Medicine, 40, 565-600. https://doi.org/10.2165/11531930-000000000-00000

Yang, X., Telama, R., Hirvensalo, M., Mattson, N., Viikari, J. S. A., \& Raitakari, O. T. (2008). Longitudinal Prospective Study of Leisure-Time Physical Activity Tracking in the Metabolic Syndrome: The Cardiovascular Risk in Young Finns Study. Medicine \& Science in Sports \& Exercise, 40, 1424-1431.

https://doi.org/10.1249/MSS.0b013e318172ced4 


\section{Appendix 1}

\section{Leisure-Time PA Index, PAI, in 2007}

\section{Original questions and codes}

1) How much breathlessness and sweating do you experience when you engage in sport or physical activity?

$\begin{array}{ll}\text { Not at all } & 1 \\ \text { Moderate amount } & 2 \\ \text { A lot } & 3\end{array}$

2) How often do you engage in sport or physical activity so that you get out of breath and sweat?

$\begin{array}{ll}\text { Not at all } & 1 \\ \text { Once a month } & 1 \\ \text { Once a week } & 2 \\ 2 \text { - } 3 \text { times a week } & 2 \\ 4 \text { - } 6 \text { times a week } & 2 \\ \text { Daily } & 3\end{array}$

3) How many hours per week do you usually engage in sport or physical activity so that you get out of breath and sweat?

$\begin{array}{ll}\text { Not at all } & 1 \\ \text { About } 1 / 2 \text { hour } & 1 \\ \text { About } 1 \text { hour } & 1 \\ \text { About } 2 \text { - } 3 \text { hours } & 2 \\ \text { About } 4 \text { - 6 hours } & 2 \\ 7 \text { hours or more } & 3\end{array}$

4) How much time do you usually spend in one session of sport of physical activity?

$\begin{array}{ll}\text { Less than } 20 \text { minutes } & 1 \\ 20-40 \text { minutes } & 2 \\ 40-60 \text { minutes } & 2 \\ \text { More than } 60 \text { minutes } & 3\end{array}$

5) Do you participate in organized physical activity (e.g. in sport club)?

Not at all

Occasionally

1

Regularly, about once per week 2

Several hours and times per week 3

Leisure-time PA index PAI total, range 5 - 15

\section{Physical Activity Expressed as METh/Wk Values}

\section{Original questions and coefficients}

1) Leisure-time $P A$ as $M E T h / w k$ values

Intensity: How much breathlessness and sweating do you experience when you engage in physical activity or sport?

Not at all 2 


\section{Moderate 4}

A lot 8

Frequency: How often do you engage in sport or physical activity so that you get out of breath and sweat?

$\begin{array}{ll}\text { Not at all } & 0 \\ \text { Once a month } & 0.25 \\ \text { Once a week } & 1 \\ 2 \text { - } 3 \text { times a week } & 2.5 \\ 4 \text { - } 6 \text { times a week } & 5 \\ \text { Daily } & 7\end{array}$

Duration: How much time do you usually spend ona physical activity session?

Less than 20 minutes $\quad 0.33$

20 - 40 minutes $\quad 0.5$

40 - 60 minutes $\quad 0.83$

More than 60 minutes $\quad 1.33$

Leisure-time PA, METh/wk $=$ Intensity $\times$ Frequency $\times$ Duration

\section{Commuting PA as METh/Wk Values}

How do you usually commute or travel to work daily?

$\begin{array}{clll}\text { summer } & \text { winter } & \\ \square & \square & \text { personal car or carpool } & \\ \square & \square & \text { public transport } & \mathrm{km} \\ \square & \square & \text { on foot } & \mathrm{km} \\ \square & \square & \text { by bicycle } & -\mathrm{km} \\ \mathrm{km}\end{array}$

Distance travelled was converted into minutes using average speed for walking $5 \mathrm{~km} / \mathrm{h}$ and for biking $15 \mathrm{~km} / \mathrm{h}$, walking to and from bus was also taken into account.

\section{Habitual PA as METh/Wk Values}

How many hours do you spend time on the following activities per month?

Heavy yard and habitual work? hours

Moderately heavy yard and habitual work? hours

Light yard and habitual work? hours

Hours were converted into METh/wk values by dividing monthly hours with 4.3 and then multiplying with the intensity values of 4 MET for heavy activities, 3 MET for moderate activities and 2 for light activities.

\section{Total PA as METh/Wk Values}

A sum of leisure-time PA METh/wk, commuting PA METh/wk, and habitual PA METh/wk. 